\title{
DIFICULTADES INTERPERSONALES EN LA ADOLESCENCIA: ¿FACTOR DE RIESGO DE FOBIA SOCIAL?
}

\author{
CÁNDIDO J. INGLÉS, F. XAVIER MÉNDEZ y M. DOLORES HIDALGO \\ Universidad de Murcia
}

(Aceptado en junio de 2001)

\begin{abstract}
El principal objetivo de este estudio fue analizar la relación de las dificultades interpersonales en la adolescencia, por un lado, con la fobia social (generalizada y no generalizada) y, por otro, con variables de personalidad (introversión y neuroticismo). Se recluto una muestra de 538 escolares, de 12 a 18 años, que cumplimentaron la siguiente batería de autoinformes: Cuestionario de Evaluación de Dificultades Interpersonales en la Adolescencia, Inventario de Ansiedad y Fobia Social, Cuestionario de Confianza para Hablar en Público (versión abreviada de 12 items), y Cuestionario de Personalidad de Eysenck. Los adolescentes con fobia social generalizada, miedo a hablar en público, introvertidos e inestables emocionales, informaron más dificultades interpersonales que los adolescentes sin fobia social generalizada, sin miedo a hablar en público, extravertidos y estables emocionales $(p<0,001)$. La prevalencia de fobia social generalizada fue $8,18 \%$, siendo más frecuente en el género femenino que en el masculino en proporción 2:1, y alcanzando un pico en la adolescencia media (1415 años).
\end{abstract}

Palabras clave: Adolescencia, dificultades interpersonales, fobia social, introversión, miedo a hablar en público, neuroticismo.

\section{Interpersonal difficulties in adolescence: a risk factor for social phobia?}

The main aim of this study was to analyse the relationship between interpersonal difficulties in adolescence, on the one hand, with social phobia (both generalized and non-generalized), and, on the other hand, with personality variables (intraversion and neuroticism). A sample of 538 schoolchildren between the ages of 12 to 18 were recruited. They then filled in the following set of self-reports: the Questionnaire about Interpersonal Difficulties for Adolescents, the Social Phobia and Anxiety Inventory, the Personal Report of Confidence as Speaker (shortened 12-item version), and the Eysenck Personality Questionnaire. Adolescents with generalized social phobia, with fear of public speaking, introverted, and emotionally instable reported more interpersonal difficulties than adolescents without generalized social phobia, without fear of public speaking, extraverted, and emotionally stable $(p<0.001)$. The prevalence of generalized social phobia was $8.18 \%$ and was more frequent among females than among males, with a proportion of 2:1, reaching a peak in mid-adolescence (14-15 years old).

Key words: Adolescence, fear of public speaking, interpersonal difficulties, introversion, neuroticism, social phobia.

\section{INTRODUCCIÓN}

La adolescencia es un período evolutivo caracterizado por importantes cambios en las relaciones interpersonales. En pri-

Correspondencia: Cándido J. Inglés, Universidad de Murcia, Facultad de Psicología, Dpto. de Personalidad, Evaluación y Tratamiento Psicológicos, Apdo. 4021, 30008 Murcia, Fax: 968364 115, Teléfono: 968 367 722, Correo-e: jingles@um.es mer lugar, se produce una expansión de las redes extrafamiliares, de modo que el adolescente se expone a un amplio abanico de nuevas situaciones sociales (fiestas, bares, oficinas públicas, establecimientos comerciales, etc.), donde se relaciona con personas desconocidas o no allegadas (Armas y Kelley, 1989; Fereing y Lewis, 1989; Flores y Díaz, 1995). En segundo lugar, la independen- 
cia de los adultos suele acompañarse de la intensificación de las relaciones con compañeros del mismo sexo (Buhrmester y Furman, 1992; Mayseless, Wiseman y Hai, 1998), y del inicio de las relaciones románticas con el otro sexo (Bracken y Crain, 1994; Furman y Buhrmester, 1992; Hansen, Christopher y Nangle, 1992). Las relaciones con los amigos desempeñan un papel crítico en el desarrollo de habilidades sociales y de sentimientos de competencia personal, que son fundamentales para el funcionamiento apropiado en la adultez (Hansen et al., 1992; La Greca y Lopez, 1998).

Los rápidos y notables cambios en las relaciones interpersonales, y el consiguiente riesgo de aparición de dificultades y malestar, explican que la ansiedad y los miedos sociales se incrementen con la edad. Las situaciones interpersonales más temidas implican la manifestación de algún tipo de comportamiento asertivo (expresar disgusto o desacuerdo, rechazar peticiones, defender los derechos personales, etc.), con desconocidos, compañeros, especialmente del sexo opuesto, y figuras de autoridad, mientras que la actuación social más difícil es hablar en público (Beidel, 1991, Essau, Conradt y Petermann, 1999; Inderbitzen, Walters y Bukowski, 1997; Inglés, Méndez e Hidalgo, 1999, 2000; La Greca y Lopez, 1998; Walters e Inderbitzen, 1998).

El constructo dificultad interpersonal se refiere a la evaluación del propio sujeto sobre el grado de dificultad experimentado al relacionarse con diferentes clases de personas, independientemente del origen de la dificultad (déficit en aserción, emociones como ansiedad o ira, creencias irracionales). Las dificultades interpersonales tienen consecuencias negativas para el adolescente. En primer lugar, repercuten en el rendimiento académico debido a la escasa participación en clase, a la resistencia a presentar tra- bajos en público y a la tendencia a evitar preguntar al profesor impidiendo la aclaración de dudas (Beidel, 1991; Francis y Radka, 1995; Lawrence y Bennet, 1992). En segundo lugar, la evitación de las relaciones con los compañeros genera aislamiento y sentimientos de soledad (Inderbitzen, Clark y Solano, 1992; Inderbitzen et al., 1997; Walters e Inderbitzen, 1998), y la correspondiente baja tasa de reforzamiento social origina depresión (Francis, Last y Strauss, 1992). Finalmente, los déficit de habilidades sociales concomitantes conducen a una relación disfuncional con los padres, que impide la comunicación, negociación y resolución de conflictos, efectivas (Openshaw, Mills, Adams y Durso, 1992), y aumenta el riesgo de consumo de drogas legales e ilegales (Clark y Kirisci, 1996; Clark y Sayette, 1993). Por tanto, la investigación de los factores que inhiben o interfieren las relaciones interpersonales del adolescente es relevante en el ámbito de la psicopatología del desarrollo.

El objetivo principal de este estudio fue analizar la relación de las dificultades interpersonales en la adolescencia, con la fobia social, tanto generalizada como no generalizada, y con variables de personalidad. Existen diferencias individuales en la predisposición hacia las relaciones interpersonales. Por un lado, la mayor tendencia a la evitación social de los sujetos introvertidos se traduce en menos experiencias de aprendizaje y, por otro, la superior labilidad de los sujetos inestables emocionales multiplica la probabilidad de experiencias de aprendizaje negativas. Ambas vías, pobreza y punitividad de las experiencias de aprendizaje, conducen a dificultades en las relaciones interpersonales y al malestar social asociado, constituyendo un factor de riesgo de la fobia social (Figura 1). 


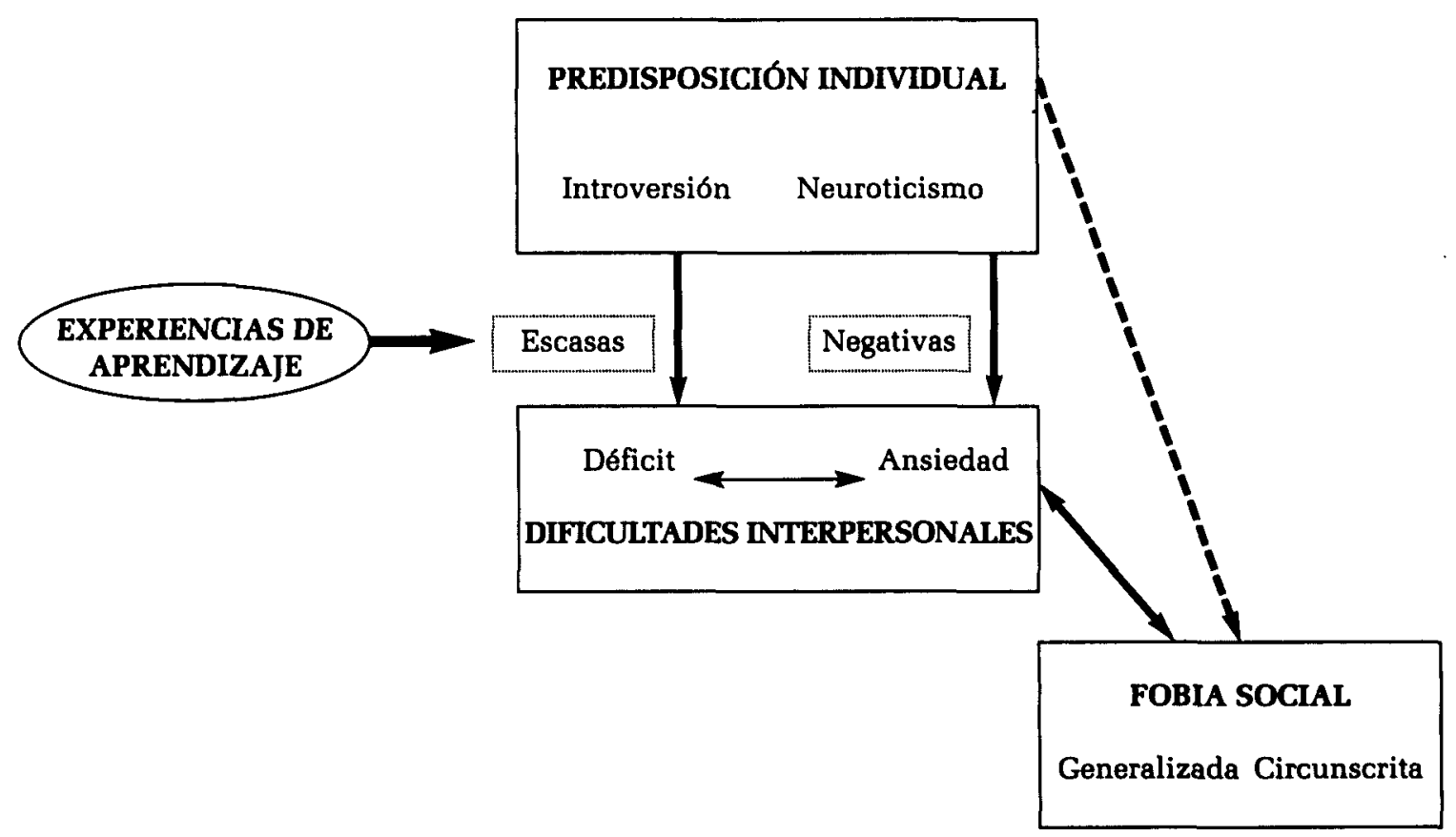

Figura 1. Relación entre variables de personalidad, dificultades interpersonales y fobia social

La investigación revela que los adolescentes con dificultades interpersonales se perciben menos asertivos (Beidel, Turner y Morris, 1995; Clark, et al., 1994; Crozier, 1995; García-López, Olivares, Hidalgo, Beidel y Turner, 2001), se valoran con menos capacidad para relacionarse con el otro sexo (Clark et al., 1994; Inglés et al., 2000; La Greca y Lopez, 1998), experimentan más miedo a hablar en público (Essau et al., 1999; Inglés et al., 1999, 2000; Martínez, Méndez, Hidalgo e Inglés, 1999), informan menos aceptación y apoyo de su grupo de compañeros (Inderbitzen et al., 1997; Walters e Inderbitzen, 1998), y presentan relaciones más conflictivas con los padres (Openshaw et al., 1992). Por otro lado, también se ha hallado con muestras adolescentes que las dificultades interpersonales correlacionan positivamente con introversión y neuroticismo (Furnham, 1984; Furnham y Gunter, 1983; Gismero, 2000; Rolf, 1972; Silva y Martorell, 1987; Young y Bradley, 1998). Por tanto, se plantean las hipótesis de que los adolescentes con fobia social generalizada, miedo a hablar en público, introvertidos, inestables emocionales, presentan mayores dificultades interpersonales que los adolescentes sin fobia social generalizada, sin miedo a hablar en público, extravertidos, estables emocionales.

Los datos sobre prevalencia de fobia social generalizada en la adolescencia varían según los criterios diagnósticos utilizados, las fuentes de información recabadas, etc. Usando los criterios del DSM-IV sendos estudios con adolescentes alemanes obtuvieron índices del 1,6\% (Essau et al., 1999) y del 4,0\% (Wittchen, Stein, y Kessler, 1999). Un estudio epidemiológico holandés basado en los criterios del DSM-III-R halló $3,7 \%$ y $6,3 \%$, cuando los informantes eran los adolescentes y los padres respectivamente (Verhulst, van der Ende, Ferdinand y Kasius, 1997). Los valores son similares en población adolescente española, encontrándose $1,7 \%$ según los criterios del DSM-III-R y 5,5\% según los criterios de la ICD-10 (Canals, Domènech, Carbajo y 
Blade, 1997). Existe evidencia sobre mayor prevalencia de fobia social en las chicas que en los chicos (Clark et al., 1994; Essau et al., 1999; La Greca y Lopez, 1998; Olivares, García-López, Hidalgo, Turner y Beidel, 1999b), y sobre su inicio en la infancia tardía y preadolescencia alcanzando un pico en la adolescencia media (Essau et al., 1999; Schneier, Johnson, Horning, Liebowitz y Weissman, 1992; Wittchen, Essau, von Zerssen, Krieg y Zaudig, 1992). Así pues, un objetivo secundario de esta investigación fue estimar la prevalencia de fobia social generalizada en población adolescente española y analizar su distribución por género y edad.

\section{MÉTODO}

\section{Sujetos}

Se seleccionaron aleatoriamente 559 estudiantes en dos colegios, uno público y otro privado, de la ciudad de Murcia, de los que $21(3,76 \%)$ fueron excluidos por omisiones o errores en sus respuestas, por no alcanzar un nivel satisfactorio en la escala de Sinceridad del Cuestionario de Personalidad de Eysenck, o por no obtener por escrito el consentimiento informado de los padres para participar en la investigación. La muestra se compuso de 538 adolescentes, con un rango de edad de 12 a 18 años $(M=14,33 ; D T=$ 1,74). La Tabla 1 muestra la distribución de los sujetos por género y edad.

Por medio de la prueba $\chi^{2}$ de homogeneidad de la distribución de frecuencias, se comprobó que no existían diferencias significativas entre los seis grupos de género $x$ edad, $\chi^{2}=3,65 ; p=0,16$. El tamaño del efecto $(P h i=0,08)$ confirmó que la diferencia fue insignificante.

\section{Instrumentos}

- Cuestionario de Evaluación de Dificultades Interpersonales en la Adolescencia (CEDIA; Inglés et al., 2000). Es un autoinforme para adolescentes de 12 a 18 años, con sendas formas para el género masculino y femenino. Las preguntas muestrean una amplia gama de relaciones interpersonales con personas de diferente edad, género, nivel de autoridad y grado de conocimiento o confianza, en diversos contextos, hogar, colegio, compañeros del mismo o de distinto sexo, y situaciones de calle, comerciales o de servicios. El sujeto valora su grado de dificultad de acuerdo con una escala Likert de cinco puntos $(0=$ ninguna dificultad; 4 = máxima dificultad). Inglés, Hidalgo y Méndez (2001) aplicaron el cuestionario resultante del estudio piloto a una muestra representativa de 4.240 escolares, de 12 a 18 años, que redujo los 40 items a 36 , agrupados en cinco factores, corroborados por un análisis factorial confirmatorio mediante validación cruzada: Aserción, Sexo Opuesto, Hablar en Público, Familia y Amigos, que explicaron el $42,86 \%$ de la varianza. La consistencia interna $(0,90)$ y la fiabilidad test-retest $(0,78)$, con un intervalo de dos semanas, fueron adecuadas.

Tabla 1. Número (y porcentaje) de sujetos de la muestra clasificados por género y grupos de edad

\begin{tabular}{lcrcc}
\hline & $12-13$ años & $14-15$ años & $16-18$ años & Total \\
\hline Varones & $187(34,76 \%)$ & $97(18,03 \%)$ & $38(7,06 \%)$ & $322(59,85 \%)$ \\
Mujeres & $115(21,38 \%)$ & $63(11,71 \%)$ & $38(7,06 \%)$ & $216(40,15 \%)$ \\
Total & $302(56,13 \%)$ & $160(29,74 \%)$ & $76(14,12 \%)$ & $538(100 \%)$ \\
\hline
\end{tabular}


- Social Phobia and Anxiety Inventory (SPAI; Turner, Beidel, Dancu y Stanley, 1989). Se utilizó únicamente la escala Fobia Social, debido a que varios estudios han mostrado que es el índice más adecuado de dicho trastorno (García-López et al., 2001; Olivares, GarcíaLópez, Hidalgo, Turner y Beidel, 1999a). Los 32 items evalúan la frecuencia de las respuestas de ansiedad provocadas por diferentes situaciones sociales con desconocidos, figuras de autoridad, miembros del sexo contrario y gente en general, mediante una escala Likert de siete puntos ( $1=$ nun$c a ; 7=$ siempre). Clark et al. (1994) hallaron un coeficiente alfa de 0,97 con adolescentes anglo-parlantes y Olivares et al. (1999b) obtuvieron un valor similar $(0,95)$ con adolescentes hispano-parlantes. La fiabilidad testretest $(0,86)$, para un período de dos semanas, fue adecuada (García-López et al., 2001). La validez de constructo, estudiada mediante la correlación con medidas de fobia social, ansiedad y aserción fue satisfactoria con adolescentes anglo e hispano-parlantes.

- Personal Report of Confidence as Speaker (PRCS; Paul, 1966). Es un autoinforme de 30 items, la mitad positivos y la mitad negativos, desarrollado a partir de un banco inicial de 104 items (Gilkinson, 1942). Evalúa las respuestas afectivas, cognitivas y conductuales que el sujeto experimenta durante la impartición de una charla. Se administró la versión abreviada de 12 items (PRCS-12), propuesta por Méndez, Inglés e Hidalgo (1999), que se compone de seis items positivos y seis negativos, valorados con una escala Likert de seis puntos $(1=$ totalmente de acuerdo; 6 = totalmente en desacuerdo). La puntuación mínima es 12 y la máxima 72, siendo 42 el valor central. Se corrige invirtiendo la puntuación de los items positivos y sumándola a la de los items negativos, de modo que a mayor puntuación menos confianza para hablar en público. Martínez et al. (1999) analizaron las propiedades psicométricas con una muestra de 788 adolescentes, de 12 a 17 años. El análisis factorial confirmatorio apoyó la existencia de dos dimensiones: Confianza para Hablar en Público y Miedo a Hablar en Público. La consistencia interna $(\alpha=0,84)$ y la fiabilidad testretest $(r=0,81)$ fueron adecuadas. El coeficiente de correlación de Pearson entre las versiones de 30 y 12 items fue elevado $(r=0,87)$ y estadísticamente significativo $(p<0,001)$.

- Eysenck Personality Questionnaire (EPQ; Eysenck y Eysenck, 1975). Es uno de los autoinformes más utilizados en evaluación de la personalidad. Incluye tres dimensiones: Introversión, Neuroticismo, y Psicoticismo, más una medida adicional de Sinceridad. Existen dos formas: el EPQ-J (81 items) para niños y adolescentes de 8 a 15 años y el EPQ-A (90 items) para jóvenes a partir de 16 años y adultos. El formato de respuesta es dicotómico (Sí/No). La consistencia interna para población española fue de 0,65 a 0,82 (EPQ-J) y de 0,70 a 0,85 (EPQ-A) (Eysenck y Seisdedos, 1978). La fiabilidad test-retest, para un período de seis meses en población británica fue de 0,61 a 0,88 (EPQ-J) $y$ de 0,71 a 0,90 (EPQ-A) (Eysenck y Eysenck, 1975). La validez de constructo fue analizada mediante correlaciones con autoinformes de adaptación, inteligencia y depresión, hallándose índices aceptables. En el presente estudio no se administró la escala Psicoticismo.

\section{Procedimiento}

Se llevó a cabo una entrevista con los directores y los jefes de los departamentos de orientación de los dos colegios 
participantes para exponer los objetivos de la investigación, describir los instrumentos de evaluación, solicitar permiso y promover su colaboración. Posteriormente se celebró una reunión con los padres para explicarles el estudio y solicitar el consentimiento informado por escrito autorizando a sus hijos a participar en la investigación. Los autoinformes fueron contestados colectivamente en el aula en grupos de 25 estudiantes aproximadamente. Se procedió a la entrega de los ejemplares con las instrucciones y de las hojas de respuesta para su corrección mediante ordenador. A continuación se indicó que cumplimentaran los datos de identificación y se leyó en voz alta las instrucciones, recalcando la importancia de no dejar ninguna pregunta sin contestar. Finalmente se aclararon las dudas, procurando no influir en la respuesta de los sujetos. Los investigadores estuvieron presentes durante la administración de las pruebas para proporcionar ayuda si era necesaria, para verificar la cumplimentación correcta e independiente por parte de los sujetos y para asegurar que los datos de identificación hubieran sido debidamente anotados. Para evitar la fatiga de los sujetos, los autoinformes se aplicaron en dos sesiones con al menos un día de separación dentro de la misma semana. El orden de presentación de las pruebas se estableció aleatoriamente para cada grupo de estudiantes. Los tiempos medios de aplicación fueron: 510 minutos el PRCS-12, 10-15 minutos el CEDIA, 15-20 minutos el EPQ y 20-25 minutos el SPAI.

\section{RESULTADOS}

Relación entre dificultades interpersonales y fobia social generalizada

Aplicando el punto de corte propuesto por Olivares et al. (1999a) para la escala
Fobia Social del SPAI (100; 76,57 tasa de acuerdo), la muestra $(N=538)$ se dividió en dos: a) grupo clínico: adolescentes con fobia social generalizada $\left(n_{1}=44 ; 8,18 \%\right)$, b) grupo control: adolescentes sin fobia social generalizada $\left(n_{2}=494 ; 91,82 \%\right)$. En este sentido cabe destacar que la asignación de los sujetos al grupo clínico no se estableció a partir de los datos obtenidos mediante diagnóstico clínico.

La Tabla 2 presenta los valores $t$ y la probabilidad asociada, para la puntuación total y las cinco escalas del CEDIA. Debido al tamaño muestral del estudio, la $t$ de Student puede detectar erróneamente diferencias estadísticamente significativas. Por esta razón se incluye el índice $d$ (diferencia media tipificada) propuesto por Hedges (1981), que permite valorar la magnitud o el tamaño del efecto de las diferencias. Este índice se calcula mediante el cociente entre la diferencia de las puntuaciones medias de los grupos clínico y control y el error típico de estimación. Su interpretación es sencilla: tamaño del efecto bajo $(0,20 \leq d \leq 0,50)$, moderado $(0,51 \leq d \leq 0,79)$ $\mathrm{y}$ alto $(d \geq 0,80)$.

Las dificultades interpersonales de los adolescentes con fobia social generaliza$\mathrm{da}$, fueron notablemente mayores, tanto globalmente como para hablar en público, relacionarse con el otro sexo y mostrarse asertivos. Aunque también informaron más dificultades con la familia y los amigos, las diferencias fueron de pequeño tamaño.

De acuerdo con el modelo teórico de la Figura 1, se efectuó un análisis discriminante para determinar el valor de las dificultades interpersonales para predecir la fobia social generalizada, considerando como variable criterio el diagnóstico de fobia social generalizada según el SPAI y como variable predictora la puntuación total del CEDIA. Se obtuvo una lambda de Wilks de 0,85 $(p=0,000)$, una correlación canónica de 0,39 y un coeficiente tipificado de 1 . La 
Tabla 2. Diferencias en dificultades interpersonales entre adolescentes $\sin$ y con fobia social

\begin{tabular}{lccccc}
\hline \multicolumn{1}{c}{$\begin{array}{c}\text { Dificultades } \\
\text { interpersonales }\end{array}$} & $\begin{array}{c}\text { Sin } \\
\text { fobia social }\end{array}$ & $\begin{array}{c}\text { Con } \\
\text { fobia social }\end{array}$ & \multicolumn{2}{c}{$\begin{array}{c}\text { Significación estadística } \\
\text { magnitud de las diferencias }\end{array}$} \\
\cline { 2 - 6 } & $M(D T)$ & $M(D T)$ & $t_{536}$ & $p$ & $d$ \\
\hline $\begin{array}{l}\text { 1. Aserción } \\
\text { (rango: 0-64) }\end{array}$ & $15,40(8,75)$ & $24,02(9,03)$ & $-6,25$ & 0,001 & 1,02 \\
$\begin{array}{l}\text { 2. Sexo Opuesto } \\
\text { (rango: 0-28) }\end{array}$ & $11,36(6,12)$ & $20,25(4,48)$ & $-9,37$ & 0,001 & 1,47 \\
3. Hablar en Público & $5,24(3,66)$ & $10,93(5,04)$ & $-9,56$ & 0,001 & 1,50 \\
$\begin{array}{l}\text { (rango: 0-20) } \\
\text { 4. Familia } \\
\text { (rango: 0-16) }\end{array}$ & $1,52(2,04)$ & $2,43(1,52)$ & $-2,82$ & 0,01 & 0,44 \\
$\begin{array}{l}\text { 5. Amigos } \\
\text { (rango: 0-16) }\end{array}$ & $1,56(1,86)$ & $2,07(1,63)$ & $-1,75$ & 0,08 & 0,28 \\
$\begin{array}{l}\text { Total CEDIA } \\
\text { (rango: 0-144) }\end{array}$ & $35,08(16,03)$ & $59,70(16,44)$ & $-9,74$ & 0,001 & 1,53 \\
\hline
\end{tabular}

Nota . CEDIA = Cuestionario de Evaluación de Dificultades Interpersonales en la Adolescencia

variable dificultades interpersonales clasificó correctamente el $74,9 \%$ de los casos. Con el fin de identificar las dificultades interpersonales de mayor riesgo para el desarrollo de fobia social generalizada se realizó un análisis discriminante por pasos. Las dificultades para hablar en público y con el sexo opuesto predijeron la fobia social generalizada. La lambda de Wilks fue de 0,80 $(p=0,000)$, con correlación canónica de 0,45 y coeficientes tipificados de 0,62 (hablar en público) y 0,60 (sexo opuesto). Ambos factores clasificaron correctamente el $81 \%$ de los casos.

Relación entre dificultades interpersonales y miedo a hablar en público

Puesto que no se ha establecido un punto de corte del PRCS-12 para identificar adolescentes con fobia social no generalizada (miedo a hablar en público), se decidió utilizar como criterio de selección la puntuación 60 , que equivale a la media $(M=38,28)$ más dos veces la desviación típica $(D T=10,86)$. La muestra $(N=531)$ se dividió de nuevo en dos: a) grupo clínico: adolescentes con miedo a hablar en público $\left(n_{1}=17 ; 3,20 \%\right)$, b) grupo control: adolescentes sin miedo a hablar en público $\left(n_{2}=514 ; 96,80 \%\right)$. La mayor diferencia se obtuvo en dificultad para hablar en público. Los adolescentes con miedo a hablar en público presentaron también mucha más dificultad en general, en las relaciones con el otro sexo y en conducta asertiva. La diferencia en dificultad con los amigos fue mediana, mientras que en el ámbito familiar no se constató ninguna diferencia (Tabla 3).

Se llevó a cabo un análisis discriminante utilizando como variable criterio el miedo a hablar en público (PRCS-12) y como variable predictora las dificultades interpersonales (CEDIA). Se halló una lambda de Wilks de 0,94 ( $\mathrm{p}=0,000)$, una correlación canónica de 0,24 y un coeficiente tipificado de 1 . Las dificultades interpersonales clasificaron correctamente el $\mathbf{7 4 , 3} \%$ de los casos. De nuevo el análisis discriminante por pasos reveló que las dificultades para hablar en público y con el sexo opuesto predecían el miedo a hablar en público. La lambda de Wilks fue de $0,92(p=0,000)$, con correlación canónica de 0,29 y coeficientes tipificados de 0,77 (hablar en público) y 
Tabla 3. Diferencias en dificultades interpersonales entre adolescentes sin y con miedo a hablar en público

\begin{tabular}{lccccc}
\hline $\begin{array}{c}\text { Dificultades } \\
\text { interpersonales }\end{array}$ & $\begin{array}{c}\text { Sin } \\
\text { miedo a hablar } \\
\text { en público }\end{array}$ & $\begin{array}{c}\text { Con } \\
\text { miedo a hablar } \\
\text { en público }\end{array}$ & \multicolumn{2}{c}{$\begin{array}{c}\text { Significación estadística } \\
\text { y magnitud de las diferencias }\end{array}$} \\
\cline { 2 - 6 } & $M(D T)$ & $M(D T)$ & $t_{529}$ & $p$ & $d$ \\
\hline $\begin{array}{l}\text { 1. Aserción } \\
\text { (rango: 0-64) }\end{array}$ & $15,79(8,95)$ & $24,47(9,35)$ & $-3,93$ & 0,001 & 0,97 \\
$\begin{array}{l}\text { 2. Sexo Opuesto } \\
\text { (rango: 0-28) }\end{array}$ & $11,83(6,35)$ & $19,53(6,58)$ & $-4,91$ & 0,001 & 1,21 \\
$\begin{array}{l}\text { 3. Hablar en Público } \\
\text { (rango: 0-20) }\end{array}$ & $5,47(3,86)$ & $11,71(6,20)$ & $-6,39$ & 0,001 & 1,57 \\
$\begin{array}{l}\text { 4. Familia } \\
\text { (rango: 0-16) }\end{array}$ & $1,59(2,10)$ & $1,65(1,77)$ & $-0,11$ & $n . s$. & - \\
$\begin{array}{l}\text { 5. Amigos } \\
\text { (rango: 0-16) }\end{array}$ & $1,57(1,84)$ & $2,29(1,97)$ & $-2,23$ & 0,03 & 0,55 \\
$\begin{array}{l}\text { Total CEDIA } \\
\text { (rango: 0-144) }\end{array}$ & $36,26(16,96)$ & $59,94(17,44)$ & $-5,66$ & 0,001 & 1,14 \\
\hline
\end{tabular}

Nota. CEDIA = Cuestionario de Evaluación de Dificultades Interpersonales en la Adolescencia

0,40 (sexo opuesto). Ambos factores clasificaron correctamente el $79,1 \%$ de los casos.

Relación entre dificultades interpersonales e introversión

Una vez comprobado que la distribución de las puntuaciones de los sujetos del estudio se ajustaba a la distribución teórica esperada en la escala Introversión del EPQ, la muestra se dividió en tres grupos: a) extravertidos: puntuaciones superiores al centil $75(\mathrm{PD}>19)\left(n_{1}=\right.$ $116 ; 22,18 \%)$; b) controles: puntuaciones mayores que el centil 25 e iguales o menores que el centil 75 ( $14<\mathrm{PD} \leq 19)$ $\left(n_{2}=224 ; 42,83 \%\right) ;$ c) introvertidos: puntuaciones iguales o inferiores al centil 25 (PD $\leq 14)\left(n_{3}=183 ; 34,99 \%\right)$.

Los resultados de los análisis de varianza se ofrecen en la Tabla 4. Los adolescentes introvertidos presentaron más dificultades interpersonales que los extravertidos y los controles, en todas las áreas excepto en la familiar. Del mismo modo que la prueba $t$, la razón $F$ puede detectar erróneamente diferencias estadísticamente significativas en muestras grandes. En este caso, el índice $d$ (diferencia media tipificada) propuesto por Cohen (1988) permite cuantificar la magnitud de las diferencias, siendo su calculo e interpretación igual que en el caso del índice $d$ de Hedges (1981).

Los adolescentes introvertidos presentaron mucha más dificultad para hablar en público que los extravertidos $(d=1,11)$ y los controles $(d=0,89)$, y muchas más dificultades interpersonales en general que los extravertidos $(d=0,91)$.

Para conocer si la introversión predispone a experimentar dificultades interpersonales se realizó un análisis de regresión simple con la puntuación directa en dicha escala del EPQ como variable independiente y la puntuación total del CEDIA como variable dependiente. La introversión explicó el $13,7 \%$ de la varianza (coeficiente de determinación ajustado $R=0,14$ ), encontrándose una relación lineal estadísticamente significativa entre ambas variables $\left(F_{1,536}=85,28, p=0,000\right)$. El tamaño de la relación (r) fue 0,37 . Para 
coeficientes de correlación de Pearson, Cohen (1988) sugiere que valores iguales o mayores que 0,30 y menores que 0,50 indican una relación de magnitud moderada.

Por otro lado se realizaron sendos análisis discriminantes para determinar el valor predictivo de la introversión en relación con la ansiedad social. Con la fobia social generalizada como criterio se obtuvo una lambda de Wilks de 0,97 $(p=0,000)$, una correlación canónica de 0,18 y un coeficiente tipificado de 1 , clasificando correctamente el $68,2 \%$ de los casos. Con el miedo a hablar en público como criterio se halló una lambda de Wilks de 0,99 $(p=0,010)$, una correlación canónica de 0,11 y un coeficiente tipificado de 1 , clasificando correctamente el $67 \%$ de los casos.

Relación entre dificultades interpersonales y neuroticismo

Utilizando el mismo procedimiento la muestra se dividió de nuevo en tres grupos: a) inestables emocionales: puntuaciones superiores al centil 75 ( $P D>15$ ) $\left(n_{1}=164 ; 30,48 \%\right)$; b) controles: puntuaciones mayores que el centil 25 e iguales o menores que el centil $75(8<\mathrm{PD} \leq 15)$ $\left.\left(n_{2}=255 ; 47,40 \%\right) ; c\right)$ estables emocionales: puntuaciones iguales o inferiores al centil $25(\mathrm{PD} \leq 8)\left(n_{3}=119 ; 22,12 \%\right)$.

No se hallaron diferencias significativas con familiares y amigos (Tabla 5). En los restantes ámbitos las mayores diferencias se obtuvieron entre los grupos extremos, adolescentes emocionalmente inestables y estables, en dificultad para relacionarse en general $(d=1,01)$ y específicamente para hablar en público $(d=1,05)$ y mostrarse asertivo $(d=0,85)$.

Según el análisis de regresión simple la dimensión neuroticismo explicó el $11,6 \%$ de la varianza (coeficiente de determinación ajustado $R=0,11$ ), obteniéndose una relación lineal con las dificultades interpersonales estadísticamente significativa $\left(F_{1,536}=70,31, p=0,000\right)$. El tamaño de la relación $(r)$ fue medio $(0,34)$.

Los resultados de los análisis discriminantes fueron: lambda de Wilks 0,94 $(p=0,000)$, correlación canónica 0,24 , coeficiente tipificado 1 , porcentaje de casos correctamente clasificados $67,3 \%$, con fobia social generalizada, y lambda de Wilks $0,96(p=0,000)$, correlación canónica 0,20 , coeficiente tipificado 1 , porcentaje de casos correctamente clasificados $73,8 \%$, con miedo a hablar en público.

Tabla 4. Diferencias en dificultades interpersonales entre adolescentes clasificados según la dimensión Introversión

\begin{tabular}{lccccc}
\hline & Extravertidos & Controles & Introvertidos & $F_{2,520}$ & $p$ \\
\hline $\begin{array}{l}\text { 1. Aserción } \\
\text { (rango: 0-64) }\end{array}$ & $13,72(8,10)$ & $14,86(9,13)$ & $19,00(8,99)$ & 16,37 & 0,001 \\
$\begin{array}{l}\text { 2. Sexo Opuesto } \\
\text { (rango: 0-28) }\end{array}$ & $10,37(5,94)$ & $10,96(6,36)$ & $14,66(6,30)$ & 23,48 & 0,001 \\
3. Hablar en Público & $3,94(3,46)$ & $4,76(3,28)$ & $8,08(4,37)$ & 57,02 & 0,001 \\
$\quad$ (rango: 0-20) & $1,40(1,87)$ & $1,62(2,16)$ & $1,74(2,15)$ & 0,94 & n.s. \\
$\begin{array}{l}\text { 4. Familia } \\
\text { (rango: 0-16) }\end{array}$ & $1,15(1,46)$ & $1,57(1,83)$ & $1,96(2,05)$ & 7,06 & 0,001 \\
$\begin{array}{l}\text { 5. Amigos } \\
\text { (rango: 0-16) }\end{array}$ & $30,57(15,61)$ & $33,77(16,27)$ & $45,44(16,96)$ & 37,59 & 0,001 \\
$\begin{array}{l}\text { Total CEDIA } \\
\text { (rango: 0-144) }\end{array}$ & & & & & \\
\hline
\end{tabular}

Nota. CEDIA = Cuestionario de Evaluación de Dificultades Interpersonales en la Adolescencia 
Tabla 5. Diferencias en dificultades interpersonales entre adolescentes clasificados según la dimensión Neuroticismo

\begin{tabular}{lrrrrc}
\hline & Extravertidos & Controles & Introvertidos & $F_{2,520}$ & $p$ \\
\hline $\begin{array}{l}\text { 1. Aserción } \\
\text { (rango: 0-64) }\end{array}$ & $12,39(7,85)$ & $16,80(8,32)$ & $19,75(10,36)$ & 26,33 & 0,001 \\
$\begin{array}{l}\text { 2. Sexo Opuesto } \\
\text { (rango: 0-28) }\end{array}$ & $9,90(6,12)$ & $12,31(6,28)$ & $14,63(6,48)$ & 19,85 & 0,001 \\
$\begin{array}{l}\text { 3. Hablar en Público } \\
\text { (rango: 0-20) }\end{array}$ & $3,81(3,21)$ & $5,93(3,90)$ & $7,82(4,43)$ & 38,62 & 0,001 \\
$\begin{array}{l}\text { 4. Familia } \\
\text { (rango: 0-16) }\end{array}$ & $1,55(2,05)$ & $1,53(1,95)$ & $1,77(2,38)$ & 0,59 & n.s. \\
$\begin{array}{l}\text { 5. Amigos } \\
\text { (rango: 0-16) }\end{array}$ & $1,43(2,08)$ & $1,68(1,64)$ & $1,68(1,92)$ & 1,06 & n.s. \\
$\begin{array}{l}\text { Total CEDIA } \\
\text { (rango: 0-144) }\end{array}$ & $29,08(15,41)$ & $38,25(15,84)$ & $45,65(18,59)$ & 36,60 & 0,001 \\
\hline
\end{tabular}

Nota. CEDIA = Cuestionario de Evaluación de Dificultades Interpersonales en la Adolescencia

Prevalencia de la fobia social generalizada en la adolescencia

Cuarenta y cuatro adolescentes recibieron el diagnóstico de fobia social generalizada $(8,18 \%)$. La prueba $Z$ de diferencias entre proporciones detectó una prevalencia estadísticamente mayor en el género femenino $(Z=-\mathbf{2 , 3 5}$; $p=0,009$ ), siendo el doble la proporción de chicas. La fobia social generalizada es más frecuente en la adolescencia media, siendo la diferencia estadísticamente significativa con la adolescencia temprana $(Z=2,04, p=0,02)$, aunque no con la adolescencia tardía $(Z=0,93, p=0,18)$. Como se aprecia en la Tabla 6 los índices de fobia social generalizada se sitúan alrededor del ocho por cien, excepto para las chicas de 14-15 años y para los chicos de 12-13 años, en los que se obtiene la mayor y menor prevalencia puntual respectivamente.

Por otra parte, la proporción de adolescentes introvertidos con fobia social ( $n=28 ; 66,67 \%$ ) fue significativamente mayor que la de los extravertidos $(n=5$; $11,90 \%)$ y la de los controles $(n=9$; $21,43 \%), \chi^{2}=30,92 ; p=0,000$. Finalmente, la proporción de adolescentes inestables emocionales $(n=22 ; 50 \%)$ y controles ( $n=20 ; 45,45 \%)$ con fobia social fue significativamente mayor que la de los estables emocionales $(n=2$; $4,54 \%) \chi^{2}=33,55 ; p=0,000$.

\section{DISCUSIÓN}

El objetivo de este estudio fue analizar el papel de varios factores que inhiben e interfieren el funcionamiento interpersonal en la adolescencia. Como se esperaba, los adolescentes con fobia social generalizada, miedo a hablar en público, introvertidos y con tendencia a la inestabilidad emocional, experimentan mayores dificultades interpersonales. Este hallazgo es consistente con los resultados de la investigación previa.

Aunque la cuarta edición del Diagnostic and Statistical Manual of Mental Disorders define la fobia social como el "temor acusado y persistente por una o más situaciones sociales o actuaciones en público en las que el sujeto se ve expuesto a personas que no pertenecen al ámbito familiar o a la posible evaluación por parte de los demás" (American Psychiatric Association, 1994, p. 427), los adolescentes con ansiedad social también experimentan dificultades con familiares 
y amigos (La Greca y Lopez, 1998). Sin embargo, sus problemas son mayores para comportarse asertivamente, principalmente con personas desconocidas o poco allegadas, para relacionarse con miembros del otro sexo y para hablar en público, debido a que padres, hermanos $\mathrm{y}$ amigos íntimos suelen funcionar como figuras de seguridad y apoyo emocional (Volling, Youngblade y Belsky, 1997).

Respecto a la relación con variables de personalidad, los adolescentes introvertidos e inestables emocionales informan mayores dificultades interpersonales, del mismo modo que en otras investigaciones con adolescentes americanos (Rolf, 1972), británicos (Furnham, 1984; Furnham y Gunter, 1983), canadienses (Young y Bradley, 1998), y españoles (Gismero, 2000, Silva y Martorrell, 1987).

Las dificultades interpersonales son resultado de la interacción entre factores situacionales: a) tipo de conducta social (por ejemplo, aserción), b) características de la otra persona (por ejemplo, sexo opuesto), c) contexto de la relación (por ejemplo, dirigirse a un auditorio), y factores personales: a) variables de personalidad (por ejemplo, introversión) y b) emociones y preocupaciones interfirientes (por ejemplo, ansiedad social). El malestar producido por las dificultades interpersonales, y la consiguiente evitación, puede conducir con el tiempo a problemas en el área social. Varios estudios clínicos han puesto de manifiesto que alrededor del $70 \%$ de los adultos con grave inhibición social experimentan un serio deterioro en sus relaciones interpersonales (Schneier et al., 1992; Turner, Beidel, Dancu y Keys, 1986).

Nosotros planteamos la hipótesis de que las dificultades interpersonales son un factor de riesgo de fobia social generalizada y no generalizada. El rechazo y la exclusión social son importantes estresores para el adolescente (Frankel, 1990), que generan aprehensión, preocupación y malestar social, conduciendo a la evitación de las relaciones interpersonales y a la pérdida de oportunidades para una socialización adecuada (La Greca y Lopez, 1998). A su vez, la aparición de la fobia social acentúa las dificultades interpersonales. Sin embargo, la naturaleza correlacional de este estudio, impide concluir si las dificultades interpersonales son causa, correlato o consecuencia, de la fobia social.

Por otro lado, aproximadamente el ocho por cien de los adolescentes fueron diagnosticados de fobia social generalizada, índice superior al de otros estudios europeos. Este resultado puede deberse a que el diagnóstico se estableció mediante la puntuación en la escala Fobia Social del SPAI. Aunque las investigaciones con adolescentes anglo e hispano-parlantes corroboran las garantías psicométricas y la capacidad diagnóstica de esta prueba de papel y lápiz, es conveniente que en futuras investigaciones el diagnóstico no se base exclusivamente en la puntuación de corte de un autoinforme, sino que se for-

Tabla 6. Porcentaje (y frecuencia relativa) de adolescentes con fobia social generalizada según género y edad

\begin{tabular}{lcccc}
\hline & $12-13$ años & $14-15$ años & $16-18$ años & Total \\
\hline Varones & $4,81 \%$ & $7,22 \%$ & $7,89 \%$ & $5,90 \%$ \\
& $(9 / 187)$ & $(7 / 97)$ & $(3 / 38)$ & $(19 / 322)$ \\
Mujeres & $8,70 \%$ & $19,05 \%$ & $7,89 \%$ & $11,57 \%$ \\
& $(10 / 115)$ & $(12 / 63)$ & $(3 / 38)$ & $(25 / 216)$ \\
Total & $6,29 \%$ & $11,87 \%$ & $7,89 \%$ & $8,18 \%$ \\
& $(19 / 302)$ & $(19 / 160)$ & $(6 / 76)$ & $(44 / 538)$ \\
\hline
\end{tabular}


mule sobre la base de entrevistas clínicas como la Anxiety Disorders Interview Schedule for DSM-IV (ADIS-IV; Di Nardo, Brown y Barlow, 1994) y recoja información adicional de otras personas significativas como padres y compañeros. Finalmente, los resultados mostraron que la fobia social es un más frecuente en las chicas y en la adolescencia media, confirmando los hallazgos de estudios previos.

\section{REFERENCIAS BIBLIOGRÁFICAS}

American Psychiatric Association (1994). Diagnostic and statistical manual of mental disorders (4th ed). Washington, DC: Author (Barcelona: Masson, 1995).

Armas, A., y Kelly, J.A. (1989). Social relationships in adolescence: Skill development and training. En J. Worell y F. Danner (Eds.), The adolescent as decision-marker: Applications to development and education. Educational Psychology (pp. 83-109). San Diego, CA, USA: Academic Press.

Beidel, D.C. (1991). Social phobia and overanxious disorder in school-age children. Journal of the Academy of Child and Adolescent Psychiatry, 30, 545-552.

Beidel, D.C., Turner, S., y Morris, T.L. (1995). A new Inventory to assess childhood social anxiety and phobia: The Social Phobia and Anxiety Inventory for Children. Psychological Assessment, 7, 73-79.

Bracken, B.A., y Crain, R.M. (1994). Children's and adolescents' interpersonal relations: Do age, race, and gender define normalcy?. Journal of Psychoeducational Assessment, 12, 14-32.

Buhrmester, D., y Furman, W. (1992). The development of companionship and intimacy. Child Development, 58, 1101-1113.

Canals, J., Domènech, E., Carbajo, G., y Blade, J. (1997). Prevalence of DSM-III-R and ICD10 psychiatric disorders in a Spanish population of 18 year olds. Acta Psychiatrica Scandinavica, 96, 287-294.

Clark, D.B., y Kirisci, L (1996). Postraumatic stress disorder, depression, alcohol use and quality of life in adolescents. Anxiety, 2, 226-233.
Clark, D.B., y Sayette, M.A. (1993). Anxiety and the development of alcoholism: Clini$\mathrm{cal}$ and scientific issues. American Journal of Addiction, 2, 59-76.

Clark, D.B., Turner, S.M., Beidel, D.C., Donovan, J.E., Kirisci, L., y Jacob, R.G. (1994). Reliability and validity of the Social Phobia and Anxiety Inventory for Adolescents. Psychological Assessment, 6, 135-140.

Cohen, J. (1988). Statistical power analysis for the behavioral sciences (2nd Ed.). Hillsdale, NJ: Erlbaum.

Crozier, W.R. (1995). Shyness and self-esteem in middle childhood. British Journal of educational Psychology, 65, 85-95.

Di Nardo, P.A., Brown, T.A., y Barlow, D.H. (1994). Anxiety Disorders Interview Schedule for DSM-IV (Lifetime version). San Antonio, TX: Psychological Corporation.

Essau, C.A., Conradt, J., y Petermann, F. (1999). Frequency and comorbidity of social phobia and social fears in adolescents. Behaviour Research and Therapy, 37, 831-843.

Eysenck, H.J., y Eysenck, S.B.G. (1975). Manual of the Eysenck Personality Questionnaire. London: Hodder y Stoughton.

Eysenck, S.B.G., y Seisdedos, N. (1978). Estudio internacional de la personalidad. Revista de Psicología General y Aplicada, 33, 271-281.

Feiring, C., y Lewis, M. (1989). The social networks of females and males from early through middle childhood. En D. Belle (Ed.), Children's social networks and social supports (pp. 119-150). New York: Wiley.

Flores, M.F., y Díaz, R.D. (1995). Desarrollo y validación de una Escala Multidimensional de Asertividad para Estudiantes. Revista Mexicana de Psicología, 12, 133-144.

Francis, G., Last, C.G., y Strauss, C.C. (1992). Avoidant disorder and social phobia in children and adolescents. Journal of the American Academy of Child and Adolescent Psychiatry, 31, 1086-1089.

Francis, G., y Radka, D.F. (1995). Social anxiety in children and adolescents. En M. B. Stein (Ed.), Social phobia: Clinical and research perspectives (pp. 119-143). New York: Guildford Press.

Frankel, K.A. (1990). Girls' perceptions of peer relationships support and stress. Journal of Early Adolescence, 10, 69-88. 
Furman, W., y Buhrmester, D. (1992). Age and sex differences in perceptions of networks of personal relationships. Child Development, 63, 103-115.

Furnham, A. (1984). Personality, social skills, anomie and delinquency: A self-report study of a group of normal non-delinquent adolescents. Journal of Child Psychology and Psychiatry, 25, 409-420.

Furnham, A., y Gunter, B. (1983). Sex and personality differences in self-reported social skills among British adolescents. Journal of Adolescence, 6, 57-69.

García-López, L.J., Olivares, J., Hidalgo, M.D., Beidel, D.C., y Turner, S. (2001). Psychometric properties of the Social Phobia and Anxiety Inventory, the Social Anxiety Scale for Adolescents, the Fear of Negative Evaluation Scale and the Social Avoidance and Distress Scale in an adolescent Spanish-speaking sample. Journal of Psychopathology and Behavioral Assessment, 23, 51-59.

Gilkinson, H. (1942). Social fears as reported by students in college speech classes. Speech Monographs, 9, 141-160.

Gismero, E. (2000). Escala de Habilidades Sociales. Madrid: TEA.

Hansen, D.J., Christopher, J.S., y Nangle, D.W. (1992). Adolescent heterosocial interactions and dating. En V.B. Van Hasselt y M. Hersen (Eds.), Handbook of social development: A lifespan perspective (pp. 371394). New York: Plenum Press.

Hedges, L.V. (1981). Distribution theory for Glass's estimator of effect size and related estimators. Journal of Educational Statistics, 6, 107-128.

Inderbitzen, H.M., Clark, M.L., y Solano, C.H. (1992). Correlates of loneliness in midadolescence. Journal of Youth and Adolescence, 21, 151-167.

Inderbitzen, H.M., Walters K.S., y Bukowski, A.L. (1997). The role of social anxiety in adolescent peer relations: Differences among sociometric status groups and rejected subgroups. Journal of Clinical Child Psychology, 26, 338-348.

Inglés, C.J., Hidalgo, M.D., y Méndez, F.X. (2001). Interpersonal difficulties in adolescence: A new self-report. Manuscrito remitido para publicación.
Inglés, C.J., Méndez, F.X., e Hidalgo, M.D. (1999, Abril). Estudio sobre la habilidad para hablar en público en población española preuniversitaria. Comunicación presentada en el I Symposium sobre Habilidades Sociales: Técnicas y Áreas de Aplicación. Granada, España.

Inglés, C.J., Méndez, F.X., e Hidalgo, M.D. (2000). Cuestionario de Evaluación de Dificultades Interpersonales en la Adolescencia. Psicothema, 12, 390-398.

La Greca, A.M., y López, N. (1998). Social anxiety among adolescents: Linkages with peer relations and friendships. Journal of Abnormal Child Psychology, 26, 83-94.

Lawrence, B., y Bennet, S. (1992). Shyness and education: The relationship between shyness, social class and personality variables in adolescents. British Journal of Educational Psychology, 62, 257-263.

Martínez, J.A., Méndez, F.X., Hidalgo, M.D., e Inglés, C.J. (1999, Noviembre). Propiedades psicométricas de la versión abreviada del Cuestionario de Confianza para Hablar en Público. Comunicación presentada en el I Congreso Iberoamericano de Psicología Clínica y de la Salud. Granada, España.

Mayseless, O., Wiseman, H., y Hai, I. (1998). Adolescents' relationships with father, mother, and same-gender friend. Journal of Adolescent Research, 13, 101-123.

Méndez, F.X., Inglés, C.J., e Hidalgo, M.D. (1999). Propiedades psicométricas del Cuestionario de Confianza para Hablar en Público: Estudio con una muestra de alumnos de enseñanzas medias. Psicothema, 11, 65-74.

Olivares, J., García-López, L.J., Hidalgo, M.D., Turner, S., y Beidel, D.C. (1999a, Noviembre). Puntos de corte clínicos para dos medidas de ansiedad social: el Inventario de Ansiedad y Fobia Social y la Escala de Ansiedad Social para Adolescentes. Comunicación presentada en el I Congreso Iberoamericano de Psicología Clínica y de la Salud. Granada, España.

Olivares, J., García-López, L.J., Hidalgo, M. D., Turner, S.M., y Beidel, D.C. (1999b). The Social Phobia and Anxiety Inventory: Reliability and validity in an adolescent Spanish population. Journal of Psychopathology and Behavioral Assessment, 21, 67-78. 
Openshaw, D.K., Mills, T.A., Adams, G.R., y Durso, D.D. (1992). Conflict resolution in parent-adolescent dyads: The influence of social skills training. Journal of Adolescent Research, 7, 457-468.

Paul, G.L. (1966). Insight vs. desensitisation in psychotherapy. Stanford, CA: Stanford University Press.

Rolf, J. (1972). The social and academic competence of children vulnerable to schizophrenia and other behaviour pathologies. Journal of Abnormal Psychology, 80, 225-243.

Schneier, F.R., Johnson, J., Horning, C.D., Liebowitz, M.R., y Weissman, M.M. (1992). Social phobia: Comorbidity and morbidity in an epidemiology sample. Archives of General Psychiatry, 49, 282-288.

Silva, F., y Martorell, C. (1987). Bateria de Socialización (Autoevaluación). Madrid: TEA.

Turner, S.M., Beidel, D.C., Dancu, C.V., y Keys, D.J. (1986). Psychopathology of social phobia and comparison to avoidant personality disorder. Journal of Abnormal Psychology, 95, 389-394.

Turner, S.M., Beidel, D.C., Dancu, C.V., y Stanley, M.A. (1989). An empirically derived inventory to measure social fears and anxiety: The Social Phobia and Anxiety Inventory. Psychological Assessment, 1, 35-40.
Verhulst, F.C., van der Ende, J., Ferdinand, R.F., y Kasius, M.C. (1997). The prevalence of DSM-III-R diagnoses in a national sample of Dutch adolescents. Archives of General Psychiatry, 54, 329-336.

Volling, B.L., Youngblade, L.M., y Belsky, J. (1997). Young children's social relationships with siblings and friends. American Journal of Orthopsychiatry, 61, 102-111.

Walters, K.S., e Inderbitzen, H.M. (1998). Social anxiety and peer relations among adolescents: Testing a psychobiological model. Journal of Anxiety Disorders, 12, 183-198.

Wittchen, H.U., Essau, C.A., von Zerssen, D., Krieg, C., y Zaudig, M. (1992). Lifetime and six-month prevalence of mental disorders in the Munich follow-up study. European Archives of Psychiatry and Clinical Neuroscience, 241, 247-258.

Wittchen, H.U., Stein, C.A., y Kessler, R.C. (1999). Social fears and social phobia in a community sample of adolescents and young adults: Prevalence, risk factors and comorbidity. Psychological Medicine, 29, 309-323.

Young, M.R., y Bradley, M.T. (1998). Social withdrawal: Self-efficacy, happiness, and popularity in introverted and extroverted adolescents. Canadian Journal of School Psychology, 14, 21-35. 